\title{
The Longitudinal Relationship Between Satisfaction with Transitional Care and Social and Emotional Quality of Life Among Chronically Ill Adolescents
}

\author{
Jane M. Cramm • Mathilde M. H. Strating • \\ Henk M. Sonneveld • Anna P. Nieboer
}

Received: 28 August 2012 / Accepted: 16 December 2012 /

Published online: 25 January 2013

(C) The Author(s) 2013. This article is published with open access at Springerlink.com

\begin{abstract}
This study aimed to identify the relationship between satisfaction with transitional care and quality of life of chronically ill adolescents over time. This longitudinal study included adolescents with type I diabetes, juvenile idiopathic arthritis (JIA), and neuromuscular disorders (NMD). At baseline 138 respondents (response rate $31 \%$ ) filled in a questionnaire and 188 about 1 year later (response rate $43 \%$ ). Analysis of variance showed that adolescents with diabetes reported the highest physical quality of life, followed in order by those with NMD and JIA $(p \leq 0.01)$. Adolescents with diabetes reported the highest social quality of life, followed in order by those with JIA and NMD (both at $p \leq 0.001$ ). Univariate analyses showed that satisfaction with transitional care at T0 was significantly related to emotional and physical quality of life at $\mathrm{T} 1$ (both at $p \leq 0.05$ ). At $\mathrm{T} 1$, satisfaction with transitional care was significantly related to the emotional, physical, and social domains of quality of life (all at $p \leq 0.001)$. Multiple regression analyses revealed that satisfaction with transitional care at $\mathrm{T} 1$ was related to emotional $(\beta-0.20 ; p \leq 0.05)$ and social $(\beta-$ $0.35 ; p \leq 0.01)$ quality of life domains over time. This indicates that lower gap scores, which measured differences between 'best care' and 'current care,' are associated with better social and emotional quality of life in this sample of adolescents. Satisfaction with transitional care and social and emotional quality of life are related over time.
\end{abstract}

Keywords Quality of life - Adolescents - Satisfaction with care - Quality of care · Diabetes $\cdot$ Juvenile idiopathic arthritis $\cdot$ Neuromuscular disorders

J. M. Cramm • M. M. H. Strating • H. M. Sonneveld • A. P. Nieboer $(\bowtie)$

Institute of Health Policy and Management, Erasmus University, P.O. Box 1738, 3000 DR Rotterdam, The Netherlands

e-mail: nieboer@bmg.eur.nl 


\section{Introduction}

Advances in medical technology have resulted in considerable improvements in the prognosis of chronic conditions, which has increased the likelihood of chronically ill children reaching stages of adolescence and adulthood (Blum et al. 1993; Scal 2002) and in turn increased numbers of adolescents with a chronic condition (Crowley et al. 2011). This growing number of adolescents with chronic health conditions who survive into adulthood has brought the issue of transition from child to adult care to the attention.

Many adolescents with chronic health conditions experienced difficulties in the transfer to adult care and to adjust to their increased responsibility for their own care when using adult services (Anthony et al. 2009; Dovey-Pearce et al. 2005; O'Connell et al. 2003; van Staa et al. 2011a, b). This transition is a long and complex process, which occurs during an already challenging passage from adolescence to adulthood (Kelly et al. 2002; Lugasi et al. 2011; Rosen 2004). Adolescents with chronic health conditions are expected to become increasingly mature and independent, which implies a role change between parents and adolescents and between adolescents and healthcare professionals (Blum et al. 1993; Rosen 2004). As they gradually grow out of the pediatric care, chronically ill adolescents desire healthcare professionals' attitudes to become less childish, more age-appropriate, and to be treated as an equal partner in care (van Staa et al. 2011b). Health care professionals should inquire preferences of adolescents with chronic health conditions and adjust their communication style and treatment plans accordingly. In addition, research shows that there is no 'one size fits all' approach to adolescent health care. There are individual differences in preferences between adolescents with chronic health conditions (Jedeloo et al. 2010).

Transition between pediatric and adult care for adolescents with chronic health conditions is often poorly managed, with negative consequences on health and quality of life outcomes (Betz 2004; Lotstein et al. 2008; McDonagh 2005; Oeffinger et al. 2005; Reiss et al. 2005; Wojciechowski et al. 2002; Watson 2000; Wysocki et al. 1992; Yeung et al. 2008). As young people mature and their medical and personal needs change, it is important that they receive age-appropriate healthcare tailored to their medical and personal needs in order to protect their quality of life (While et al. 2004). Transition programs have been developed to bridge the gap between pediatric to adult care (Blum 1993; Scal 2002; McDonagh 2005; Reiss et al. 2005). Transition of care is defined as "the purposeful, planned movement of adolescents and young adults with chronic physical and medical conditions from child-centered to adult-oriented health care systems" (Blum et al. 1993). Satisfaction with transitional care is thus a necessary component of caring for adolescents with chronic health conditions (Binks et al. 2007; Nakhla et al. 2008; McDonagh et al. 2007). The optimal goal of transitioning from child- to adult-oriented care is to provide adolescents with uninterrupted, coordinated, developmentally appropriate, and comprehensive healthcare that addresses their medical, psychosocial, and educational/vocational needs (McDonagh et al. 2000; Blum et al. 1993). Transitional care services are expected to help smooth the process to avoid gaps in health care and maximize adult quality of life (Lotstein et al. 2009). Transitional care may consist of preparing adolescents for the transfer to adult-oriented care by formulating self- 
management goals using an individualized self-management plan or by practicing self management skills by seeing the physician without their parent being present. Also joint consultations in which the paediatrician and medical specialist from adult care together consult with the adolescent in one session may serve to prepare the adolescent for transfer.

The need for transitional care is well established for adolescents with chronic health conditions (Shaw et al. 2005, 2004a, b), but the quality of life outcomes of such care remain unclear. A limited number of studies have evaluated transitional care programs that support beneficial outcomes for patients with various chronic diseases (McDonagh 2005; Nakhla et al. 2008; Rettig and Athreya 1991; Zack et al. 2003), but most of these studies have not investigated the effects of these programs on quality of life. In the only study to date investigating quality of life impacts, McDonagh et al. (2007) found evidence that coordinated transitional care can potentially improve quality of life in adolescents with juvenile idiopathic arthritis. We do not know the longitudinal relationship between transitional care and quality of life among adolescents with other chronic conditions. The medical and healthcare fields face the challenge of assessing the quality of life and experiences of adolescents with chronic health conditions who receive transitional care, with the aim of improving quality of life in this patient population. Thus, the aim of this study was to investigate the relationship between satisfaction with transitional care and quality of life over time among adolescents with various chronic conditions.

\section{Methods}

\section{Setting and Design}

This longitudinal study was part of a larger evaluation of a collaborative Dutch quality-improvement program called 'On Your Own Feet Ahead!'. This program aimed to develop and implement innovative transitional care interventions for chronically ill adolescents. The "breakthrough method" was used as a model for improvement and implementation. Improvement teams from various organizations joined forces to achieve substantial improvements within a 1-year timeframe. Headed by a project leader, each temporary improvement team generally consisted of four to five providers from pediatric and adult care.

The improvement teams from the participating organizations were invited to attend four national conferences offering workshops and sessions in which questions could be posed to other teams or experts. Program managers provided training and instructions on the improvement methods, transferred knowledge on transitional care and acted as counsellors. The improvement teams developed and implemented their interventions under the guidance of the program managers. A toolkit with transitional care interventions was offered to each team. Examples of transitional care interventions are an individual self management plan, availability of a transition coordinator and transition protocol. Teams were invited to decide on the interventions based on their client type and the local context.

As part of a larger evaluation research, this study surveyed patients treated by teams that delivered care to adolescents with type I diabetes, juvenile idiopathic 
arthritis (JIA), and neuromuscular disorders (NMD); the latter were treated with chronic mechanical ventilation.

\section{Participants and Survey Procedure}

As part of the program evaluation, adolescents treated by the teams were asked to complete questionnaires. At baseline 138 respondents (out of 441; response rate $31 \%$ ) filled in a questionnaire and 188 about 1 year later (out of 433; response rate $43 \%$ ). A total of 123 respondents filled in the questionnaire at both T0 and T1. Eligible participants were selected by the participating teams. They were 12-25-yearolds in active long-term pediatric treatment. Two exclusion criteria were applied: already transferred to adult care; and documented diagnosis of intellectual impairment. Approval for the study was obtained from the Erasmus Medical Centre Institutional Review Board. Eligible adolescents received written information and a unique access code, and were invited to complete a web-based questionnaire. Nonrespondents received a reminder by mail after 2 weeks, including a printed copy of the questionnaire. There was no financial remuneration, although participants were entered in a lottery with a change to win an iPod. At follow-up all eligible adolescents (respondents as well as non-respondents at T0) were invited to complete questionnaires. About 2 weeks later a reminder was sent. In addition, after the reminder, nonresponders were asked by telephone to complete the questionnaire.

\section{Measures}

The structured survey for adolescents asked respondents to provide background characteristics, such as age, gender, and type of chronic condition. Quality of life was assessed with the DISABKIDS condition-generic module questionnaire (Petersen et al. 2005), which consists of 37 items grouped into six dimensions: independence, physical limitation, social inclusion, social exclusion, emotion, and medication. The six dimensions were conceptually associated with three higher-order domains: emotional, physical, and social quality of life. The emotional domain incorporated the positive aspect of independent living (independence dimension), as well as all kinds of emotional reactions to having a chronic condition (emotion dimension). The physical domain included physical symptoms and limitations due to the chronic condition (physical limitation dimension), as well as the impact of taking medicine (in the forms of pills, injections, etc.; medication dimension). The social domain included aspects of stigma (social exclusion dimension) and support from friends, family, and others (social inclusion dimension) (Schmidt et al. 2006). Responses to each item were structured using a five-point Likert scale $(1=$ never, $2=$ seldom, 3 = quite often, 4 = very often, 5 = always). Domain scores were transformed linearly to a $0-100$ scale, with 100 indicating the highest quality of life. Cronbach's alpha values of the DISABKIDS condition-generic module social ( 0.80 at T0, 0.84 at T1), emotional (0.90 at T0, 0.92 at T1), and physical (0.84 at T0, 0.81 at T1) domains all indicated good reliability.

We used the 'Mind the Gap' instrument (Shaw et al. 2007, 2004c), which was developed to assess satisfaction with transitional care among adolescents with chronic health conditions, to assess adolescents' experiences with and expectations of 
transitional care. Reponses to each of the 22 items of this instrument were structured on a seven-point Likert-scale ranging from 1 (strongly disagree) to 7 (strongly agree). The items included statements about issues identified as important in earlier needs assessments (Shaw et al. 2004a, b), including management of the environment (five items), provider characteristics (11 items), and process issues (six items). Gap scores were calculated as the differences between 'best care' and 'current care' as perceived by the adolescents with no difference (score $=0$ ) indicating the absence of a discrepancy. A positive gap score indicated that 'current care' did not meet the standards of the 'expected best care,' and more positive scores indicated lower levels of satisfaction. Cronbach's alpha values confirmed the reliability of the Mind the Gap instrument (0.89 at T0, 0.88 at $\mathrm{T} 1)$.

\section{Statistical Analyses}

We investigated the longitudinal relationship between satisfaction with transitional care (Mind the Gap scores) and the emotional, physical, and social domain scores of the DISABKIDS condition-generic module instrument. Descriptive analysis included the calculation of means and standard deviations. Differences between scores at T1 and T0 were established with two-tailed paired $t$-tests, and quality of life and satisfaction with transitional care were compared among adolescents with different chronic conditions using analysis of variance. Bivariate correlations between satisfaction with care and the quality of life domains were first identified. Multiple regression analyses were then performed (among respondents who filled in questionnaires at both T0 and T1 only) to reveal significant longitudinal relationships between satisfaction with care delivery and quality of life domains after controlling for quality of life at T0, age, gender and type of chronic condition. All statistical analyses were conducted with SPSS software (ver. 17.0; SPSS, Inc., Chicago, IL, USA).

\section{Results}

At T1, $58.8 \%$ of respondents were female and their mean age was $17 \pm 1.7$ (range, $12-20)$ years. About half (53\%) of the respondents reported having diabetes, $37 \%$ had JIA, and $10 \%$ had NMD. The overall mean quality of life at T1, as measured with the DISABKIDS condition-generic module instrument, was 74.9 \pm 14.5 .

The emotional, physical and social domains of quality of life did not differ between T0 and T1. Mean gap scores, indicating perceived satisfaction with transitional care, also did not differ between T0 and T1.

Table 1 shows differences between chronic conditions in quality of life domains and satisfaction with transitional care. These results indicate that physical domain scores varied among chronic conditions at $\mathrm{T} 1\left(\mathrm{~F}_{\text {group }}=5.385 ; p \leq 0.01\right)$, but not at $\mathrm{T} 0$ $\left(\mathrm{F}_{\text {group }}=0.836 ; p=0.436\right)$. At $\mathrm{T} 1$, adolescents with diabetes reported higher physical quality of life than did those with NMD or JIA. Social domain scores varied among chronic conditions at both $\mathrm{T} 1\left(\mathrm{~F}_{\text {group }}=9.665 ; p \leq 0.001\right)$ and $\mathrm{T} 0\left(\mathrm{~F}_{\text {group }}=7.271 ; p \leq\right.$ $0.001)$. Adolescents with diabetes reported the highest social quality of life, followed in order by adolescents with JIA and those with NMD at both T0 and T1. No difference among chronic conditions was observed in emotional domain scores at 
Table 1 Differences between chronic condition in quality of life domains and satisfaction with transitional care

\begin{tabular}{|c|c|c|c|c|c|c|c|c|c|c|c|c|}
\hline & \multicolumn{6}{|l|}{$\mathrm{T} 0$} & \multicolumn{6}{|l|}{$\mathrm{T} 1$} \\
\hline & JIA & NMD & Diabetes & Total & $\mathrm{F}$ & $\mathrm{p}$ & JIA & NMD & Diabetes & Total & $\mathrm{F}$ & $\mathrm{p}$ \\
\hline \multicolumn{13}{|c|}{ Emotional quality of life } \\
\hline $\mathrm{n}$ & 31 & 12 & 72 & 115 & 0.133 & .873 & 68 & 17 & 95 & 180 & 0.881 & .416 \\
\hline mean & 78.0 & 79.0 & 76.8 & 77.4 & & & 74.8 & 76.7 & 78.2 & 76.8 & & \\
\hline sd & 16.2 & 14.5 & 16.2 & 15.9 & & & 17.6 & 16.1 & 15.2 & 16.1 & & \\
\hline \multicolumn{13}{|c|}{ Physical quality of life } \\
\hline $\mathrm{n}$ & 22 & 12 & 71 & 105 & 0.836 & .436 & 55 & 13 & 94 & 162 & 5.385 & .005 \\
\hline mean & 63.5 & 71.7 & 67.5 & 67.2 & & & 61.8 & 66.3 & 70.9 & 67.5 & & \\
\hline sd & 17.7 & 13.8 & 18.9 & 18.2 & & & 16.9 & 11.5 & 16.6 & 16.8 & & \\
\hline \multicolumn{13}{|c|}{ Social quality of life } \\
\hline $\mathrm{n}$ & 31 & 12 & 72 & 115 & 7.271 & .001 & 68 & 17 & 96 & 181 & 9.665 & $\leq .001$ \\
\hline mean & 77.5 & 67.5 & 82.1 & 79.4 & & & 76.0 & 71.2 & 83.9 & 79.8 & & \\
\hline sd & 15.4 & 11.5 & 11.5 & 13.3 & & & 16.6 & 11.2 & 12.7 & 14.9 & & \\
\hline \multicolumn{13}{|c|}{ Satisfaction with transitional care } \\
\hline $\mathrm{n}$ & 30 & 11 & 72 & 113 & 0.481 & .620 & 69 & 16 & 97 & 182 & 0.229 & .742 \\
\hline mean & 0.3 & 0.5 & 0.2 & 0.3 & & & 0.5 & 0.5 & 0.4 & 0.5 & & \\
\hline sd & 0.9 & 1.2 & 1.0 & 1.0 & & & 0.9 & 1.0 & 1.0 & 0.9 & & \\
\hline
\end{tabular}

JIA juvenile idiopathic arthritis; NMD neuromuscular disorders. A description of the quality of life and satisfaction with transitional care instruments can be found in the measures paragraph in the Methods section

T1 or T0. Similarly, satisfaction with transitional care did not differ among conditions at $\mathrm{T} 1$ or $\mathrm{T} 0$.

The results of univariate analyses showed that satisfaction with transitional care at T0 was significantly related to emotional and physical quality of life at T1 (both at $p \leq 0.05$; Table 2). Satisfaction with transitional care at T1 was significantly related to the emotional, physical and social domains of quality of life at $\mathrm{T} 1$ (all at $p \leq 0.001$ ).

Table 3 displays the multiple regression analyses. A strong longitudinal relationship was found between emotional quality of life at $\mathrm{T} 0$ and emotional quality of life at T1 $(\beta=0.69 ; p \leq 0.001)$. In addition, after controlling for emotional quality of life at T0, age, gender and chronic condition; we found a significant relationship between satisfaction with transitional care at $\mathrm{T} 1$ and emotional quality of life at T1 ( $\beta-0.20$; $p \leq 0.05$ ). We also found a strong longitudinal relationship between physical quality of life at T0 and physical quality of life at T1 $(\beta=0.56 ; p \leq 0.001)$. Adolescents with diabetes reported significantly higher physical quality of life than did adolescents with JIA $(\beta=0.27 ; p \leq 0.01)$. A strong longitudinal relationship was also found between social quality of life at T0 and social quality of life at T1 $(\beta=0.58 ; p \leq$ 0.001 ). After controlling for social quality of life at $\mathrm{T} 0$, age, gender and chronic condition, satisfaction with transitional care at $\mathrm{T} 1$ was significantly associated with social quality of life at $\mathrm{T} 1(\beta=-0.35 ; p \leq 0.01)$. This indicates that lower gap scores, 
Table 2 Associations between satisfaction with transitional care and quality of life in adolescents with chronic health conditions

\begin{tabular}{|c|c|c|c|c|c|c|}
\hline & $\begin{array}{l}\text { Emotion } \\
\text { of life at }\end{array}$ & & $\begin{array}{l}\text { Physical } \\
\text { of life at }\end{array}$ & & $\begin{array}{l}\text { Social qu } \\
\text { of life at }\end{array}$ & \\
\hline Satisfaction wi & nsitional & & & & & \\
\hline T0 Gap score & $-0.23^{*}$ & $n=81$ & $-0.25^{*}$ & $n=75$ & -0.14 & $n=81$ \\
\hline T1 Gap score & $-0.27^{* * *}$ & $n=179$ & $-0.28^{* * *}$ & $n=162$ & $-0.28^{* * *}$ & $n=180$ \\
\hline
\end{tabular}

${ }^{* * *} p \leq 0.001 ;{ }^{* *} p \leq 0.01 ;{ }^{*} p \leq 0.05$ (two-tailed)

which measured differences between 'best care' and 'current care,' are associated with better social and emotional quality of life in this sample of adolescents.

\section{Discussion}

This study evaluated the longitudinal relationship between satisfaction with transitional care and the emotional, physical, and social domains of quality of life among adolescents with diabetes, JIA, and NMD. Adolescents with diabetes had the highest social and physical quality of life outcomes, with JIA reported the lowest physical quality of life outcomes, and those with an NMD reported the lowest social quality of

Table 3 Longitudinal relationship with quality of life as assessed by multiple regression analyses

\begin{tabular}{lccc}
\hline & $\begin{array}{l}\text { Emotional quality } \\
\text { of life at T1 }(n=80)\end{array}$ & $\begin{array}{l}\text { Physical quality } \\
\text { of life at T1 }(n=72)\end{array}$ & $\begin{array}{l}\text { Social quality } \\
\text { of life at T1 }(n=80)\end{array}$ \\
\hline $\begin{array}{l}\text { Quality of life at T0 } \\
\text { Background characteristics }\end{array}$ & $0.69^{* * *}$ & $0.56^{* * *}$ & $0.58^{* * *}$ \\
Age & 0.01 & 0.01 & 0.05 \\
Gender (Male) & 0.03 & -0.02 & 0.03 \\
Chronic condition & 0.01 & $0.27^{* *}$ & 0.03 \\
Diabetes & 0.00 & 0.15 & 0.01 \\
Neuromuscular disorder & & & 0.18 \\
Satisfaction with transitional care & 0.10 & -0.07 & $-0.35^{* *}$ \\
T0 Gap score & $-0.20^{*}$ & -0.10 & $39.8 \%$ \\
T1 Gap score & $51.7 \%$ & $42.7 \%$ & 7.306 \\
Adjusted R & 13.252 & 8.664 & \\
F & & & \\
\hline
\end{tabular}

${ }^{* * *} p \leq 0.001 ;{ }^{* *} p \leq 0.01 ;{ }^{*} p \leq 0.05$ (two-tailed). Juvenile rheumatoid arthritis is the reference group of the chronic conditions. Listwise deletion of missing cases was used for the analyses. Findings are based on respondents who filled in the questionnaire at both $\mathrm{T} 0$ and $\mathrm{T} 1$ only

${ }^{\text {a }}$ Emotional quality of life at T0 was used as independent variable to asses the longitudinal relationship with emotional quality of life at T1. Physical quality of life at T0 was used as independent variable to asses the longitudinal relationship with physical quality of life at T1. Social quality of life at T0 was used as independent variable to asses the longitudinal relationship with social quality of life at T1 
life outcomes. No difference in emotional quality of life was found among adolescents with these chronic conditions. The overall mean quality of life, as measured with the DISABKIDS condition-generic module instrument at T0 (74.4 \pm 14.1$)$ and T1 (74.9 \pm 14.5$)$, were comparable to the findings of Schmidt et al. (2006), who used the same instrument to measure overall quality of life $(76.9 \pm 14.8)$ among adolescents in a variety of countries with various chronic conditions.

Whereas univariate analyses revealed that satisfaction with transitional care was related to all three quality of life domains, multiple regression analyses only demonstrated a significant relationship between satisfaction with transitional care and social and emotional quality of life over time. Satisfaction with transitional care included process aspects such as the responsibility of a particular staff member for coordinating each adolescent's care, the availability of a staff member to provide information about other people/organizations that could provide support (e.g., specialist career counselor, benefit advisor, support group), and staff members help planning for the future and prepare for the transition from child- to adult-oriented care. But also management of the environment (e.g. provide opportunities to meet other young people with the same chronic condition and display relevant health-related information in waiting rooms) and provider characteristics (e.g. availability of staff who understand the realities of being a teenager and who know how to talk and listen to teenagers) are important aspects of satisfaction among adolescents with chronic health conditions with transitional care (Dovey-Pearce et al. 2005; Shaw et al. 2007, 2004c; van Staa et al. 2011b). Hospitals and rehabilitation centers that provide care to adolescents with chronic health conditions should thus pay attention to these issues and try to implement interventions that aim to improve them. By implementing interventions such as individual self management plans and improving provider characteristics by training them in motivational interviewing may contribute to a better match between adolescents' needs and the care they receive. Furthermore, individualized self-management or transition plans are expected to stimulate adolescents to become more independent and to enhance their self-management and coping skills not only with respect to the physical aspects of their condition, but also with respect to the psychosocial aspects. Transitional care needs to better address the changing needs and roles of adolescents and their families. Interventions with a comprehensive focus on adolescents' needs and not just physical functioning only, are expected to improve social and emotional quality of life outcomes among adolescents with chronic health conditions.

Some limitations should be taken into account when interpreting our study findings. First, our group of adolescents with NMD was smaller than our other groups. Although our results are largely in agreement with those of other studies, they should be interpreted with caution due to this imbalance in our study sample. Second, although the response rate of 31 and $43 \%$ might seem low, and there is the potential danger of non-response bias, it is similar compared to other studies in which the respondents also received a questionnaire by mail (Picavet 2001; Buttle and Thomas 1997). Finally, since this was not a randomized control study we found significant relationships between transitional care and social and emotional quality of life over time only, we could not identify the predictive nature of transitional care on social and emotional quality of life. 
Strength of this study is that we investigated the longitudinal relationship between satisfaction with transitional care among adolescents with a variety of chronic conditions, while controlling for quality of life at baseline.

We conclude that greater satisfaction with transitional care is significantly related to better social and emotional quality of life among adolescents with diabetes, JIA, and NMD over time. Thus, the improvement of adolescents' transitional care in hospitals and rehabilitation centers is expected to be beneficial for their quality of life.

Acknowledgments This study is funded with a grant from the Netherlands organisation for health research and development (ZonMw) grant number: 157002014. The views are those of the authors.

Conflict of Interest We declare no conflict of interest.

Open Access This article is distributed under the terms of the Creative Commons Attribution License which permits any use, distribution, and reproduction in any medium, provided the original author(s) and the source are credited.

\section{References}

Anthony, S. J., Martin, K., Drabble, A., Seifert-Hansen, M., Dipchand, A. I., \& Kaufman, M. (2009). Perceptions of transitional care needs and experiences in pediatric heart transplant recipients. American Journal of Transplantation, 9, 614-619.

Betz, C. L. (2004). Transition of adolescents with special health care needs: review and analysis of the literature. Issues in Comprehensive Pediatric Nursing, 27, 179-241.

Binks, J. A., Barden, W. S., Burke, T. A., \& Young, N. L. (2007). What do we really know about the transition to adult-centered health care? A focus on cerebral palsy and spina bifida. Archives of Physical and Medical Rehabilitation, 88, 1064-1073.

Blum, R. W. M., Garell, D., Hodgman, C. H., Jorissen, T. W., Okinow, N. A., Orr, D. P., et al. (1993). Transition from child-centered to adult health-care systems for adolescents with chronic conditions. Journal of Adolescent Health, 14, 570-576.

Buttle, F., \& Thomas, G. (1997). Questionnaire colour and mail survey response rate. Journal of the Market Research Society, 39, 625-626.

Crowley, R., Wolfe, I., Lock, K., \& McKee, M. (2011). Improving the transition between paediatric and adult healthcare: a systematic review. Archives of Disease in Childhood. doi:10.1136/ adc. 2010.202473 .

Dovey-Pearce, G., Hurrell, R., May, C., Walker, C., \& Doherty, Y. (2005). Young adults' (16-25 years) suggestions for providing developmentally appropriate diabetes services: a qualitative study. Health \& Social Care in the Community, 13, 409-419.

Jedeloo, S., van Staa, A., Latour, J. M., \& van Exel, N. J. (2010). Preferences for health care and selfmanagement among Dutch adolescents with chronic conditions: a Q-methodological investigation. International Journal of Nursing Studies, 47, 593-603.

Kelly, A. M., Kratz, B., Bielski, M., \& Rinehart, P. M. (2002). Implementing transitions for youth with complex chronic conditions using the medical home model. Pediatrics, 110, 1322-1327.

Lotstein, D. S., Inkelas, M., Hays, R. D., Halfon, N., \& Brook, R. (2008). Access to care for youth with special health care needs in the transition to adulthood. Journal of Adolescent Health, 43, 23-29.

Lotstein, D. S., Ghandour, R., Cash, A., McGuire, E., Strickland, B., \& Newacheck, P. (2009). Planning for health care transitions: results from the 2005-2006 National Survey of Children with special health care needs. Pediatrics, 123, 145-152.

Lugasi, T., Achille, M., \& Stevenson, M. (2011). Patients' perspective on factors that facilitate transition from child-centered to adult-centered health care: a theory integrated metasummary of quantitative and qualitative Studies. Journal of Adolescent Health, 48, 429-440.

McDonagh, J. E. (2005). Growing up and moving on: transition from pediatric to adult care. Pediatric Transplantation, 9, 364-372. 
McDonagh, J. E., Foster, H., Hall, M. A., Chamberlain, M. A., \& on behalf of the British Paediatric Rheumatology Group. (2000). Audit of rheumatology services for adolescents and young adults in the UK. Rheumatology, 39, 596-602.

McDonagh, J. E., Southwood, T. R., \& Shaw, K. L. (2007). British Society of Paediatric and Adolescent Rheumatology: the impact of a coordinated transitional care programme on adolescents with juvenile idiopathic arthritis. Rheumatology, 46, 161-168.

Nakhla, M., Daneman, D., Frank, M., \& Guttmann, A. (2008). Translating transition: a critical review of the diabetes literature. Journal of Pediatric Endocrinology \& Metabolism, 21, 507516.

O’Connell, B., Bailey, S., \& Pearce, J. (2003). Straddling the pathway from paediatrician to mainstream health care: transition issues experienced in disability care. Australian Journal of Rural Health, 11, 5763.

Oeffinger, K. C., Mertens, A. C., Hudson, M. M., Gurney, J. G., Casillas, J., Chen H., Whitton, J., Yeazel, M., Yasui, Y., \& Robison, L. L. (2004). Health care of young adult survivors of childhood cancer: a report from the Childhood Cancer Survivor Study. Ann Fam Med, 2, 61-70.

Petersen, C., Schmidt, S., Power, M., Bullinger, M., \& the DISABKIDS Group (2005). Development and pilot-testing of a health-related quality of life chronic generic module for children and adolescents with chronic health conditions: a European perspective. Quality of Life Research, 14, 1065-1077.

Picavet, H. S. J. (2001). National health surveys by mail or home interview. Effects on response. Journal of Epidemiology and Community Health, 55, 408-413.

Reiss, J. G., Gibson, R. W., \& Walker, L. R. (2005). Health care transition: youth, family, and provider perspectives. Pediatrics, 115, 112-120.

Rettig, P., \& Athreya, B. H. (1991). Adolescents with chronic disease. Transition to adult health care. Arthritis Care and Research, 4, 174-180.

Rosen, D. S. (2004). Transition of young people with respiratory diseases to adult health care. Paediatric Respiratory Review, 5, 124-131.

Scal, P. (2002). Transition for youth with chronic conditions: primary care physicians' approaches. Pediatrics, 110, 1315-1321.

Schmidt, S., Debensason, D., Mühlan, H., Petersen, C., Power, M., Simeoni, M. C., et al. (2006). The DISABKIDS generic quality of life instrument showed cross-cultural validity. Journal of Clinical Epidemiology, 59, 587-598.

Shaw, K. L., Southwood, T. R., \& McDonagh, J. E. (2004a). Users' perspectives of transitional care for adolescents with juvenile idiopathic arthritis. Rheumatology, 43, 770-778.

Shaw, K. L., Southwood, T. R., \& McDonagh, J. E. (2004b). Developing a programme of transitional care for adolescents with juvenile idiopathic arthritis: results of a postal survey. Rheumatology, 43, 211219 .

Shaw, K. L., Southwood, T. R., \& McDonagh, J. E. (2004c). Transitional care for adolescents with juvenile idiopathic arthritis: results of a Delphi study. Rheumatology, 43, 1000-1006.

Shaw, K. L., Southwood, T. R., McDonagh, J. E., \& on behalf of the British Society of Paediatric and Adolescent Rheumatology. (2005). Growing up and moving on in rheumatology: a multicentre cohort of adolescents with juvenile idiopathic arthritis. Rheumatology, 44, 806-812.

Shaw, K. L., Southwood, T. R., \& McDonagh, J. E. (2007). Development and preliminary validation of the 'Mind the Gap' scale to assess satisfaction with transitional care among adolescents with juvenile idiopathic arthritis. Child: Care, Health and Development, 33, 280388.

van Staa, A. L., Jedeloo, S., van Meeteren, J., \& Latour, J. M. (2011a). Crossing the transition chasm: experiences and recommendations for improving transitional care of young adults, parents and providers. Child: Care, Health and Development, 37, 821-832.

van Staa, A., Jedeloo, S., \& van der Stege, H. (2011b). "What we want": chronically ill adolescents' preferences and priorities for improving health care. Patient Prefer Adherence, 5, 291305.

Watson, A. R. (2000). Non-compliance and transfer from paediatric to adult transplant unit. Pediatric Nephrology, 14, 469-472.

While, A., Forbes, A., Ullman, R., Lewis, S., Mathes, L., \& Griffiths, P. (2004). Good practices that address continuity during transition from child to adult care: synthesis of the evidence. Child: Care, Health and Development, 30, 439-452.

Wojciechowski, E. A., Hurtig, A., \& Dorn, L. (2002). A natural history study of adolescents and young adults with sickle cell disease as they transfer to adult care: a need for case management services. Journal of Pediatric Nursing, 17, 18-27. 
Wysocki, T., Hough, B. S., Ward, K. M., \& Green, L. B. (1992). Diabetes mellitus in the transition to adulthood: adjustment, self-care, and health status. Journal of Developmental and Behavioral Pediatrics, 13, 194-201.

Yeung, E., Kay, J., Roosevelt, G. E., Brandon, M., \& Yetman, A. T. (2008). Lapse of care as a predictor for morbidity in adults with congenital heart disease. International Journal of Cardiology, 125, 62-65.

Zack, J., Jacobs, C. P., Keenan, P. M., Harney, K., Woods, E. R., Colin, A. A., et al. (2003). Perspectives of patients with cystic fibrosis on preventive counselling and transition to adult care. Pediatric Pulmonology, 36, 376-383. 